\title{
Securing Highly-Sensitive Information in Smart Mobile Devices through Difficult-to-Mimic and Single-Time Usage Analytics
}

\author{
Saiyma Sarmin \\ University of Colorado Boulder \\ saiyma.sarmin@colorado.edu
}

\author{
Nafisa Anzum \\ University of Waterloo \\ nanzum@uwaterloo.ca
}

\author{
Kazi Hasan Zubaer \\ Google, UK \\ kazizubaer@google.com
}

\author{
Farzana Rahman \\ Florida International University \\ farahman@fiu.edu
}

\author{
A. B. M. Alim Al Islam \\ Bangladesh University of Engineering \\ and Technology \\ alim_razi@cse.buet.ac.bd
}

\begin{abstract}
The ability of smart devices to recognize their owners or valid users gains attention with the advent of widespread highly sensitive usage of these devices such as storing secret and personal information. Unlike the existing techniques, in this paper, we propose a very lightweight single-time user identification technique that can ensure a unique authentication by presenting a system nearto-impossible to breach for intruders. Here, we have conducted a thorough study over single-time usage data collected from 33 users. The study reveals several new findings, which in turn, leads us to a novel solution exploiting a new machine learning technique. Our evaluation confirms that the proposed solution operates with only 5\% False Acceptance Rate (FAR) and only 6\% False Rejection Rate (FRR) over the data collected from 33 users. We further evaluate the performance through comparing its performance with some existing machine learning techniques. Finally, we perform a real implementation of our proposed solution as a mobile application to conduct a rigorous user evaluation over 27 participants using three different devices in order to show how the solution works in practical situations. Outcomes of the user evaluation demonstrate as low as $0 \%$ FAR after letting intruders to mimic the actual user, which ensures extremely low probability of being breached. Moreover, we let 2 users to continuously use our application over 25 days in different states during their operation. Outcomes of this evaluation demonstrate as low as $1 \%$ FRR confirming the usability of our technique in long-term usage.
\end{abstract}

\section{CCS CONCEPTS}

- Human-centered computing $\rightarrow$ Mobile computing;

\section{KEYWORDS}

Smartphone, Behavioral biometrics, Security, Mean-SD Clustering

Permission to make digital or hard copies of all or part of this work for personal or classroom use is granted without fee provided that copies are not made or distributed for profit or commercial advantage and that copies bear this notice and the full citation on the first page. Copyrights for components of this work owned by others than ACM must be honored. Abstracting with credit is permitted. To copy otherwise, or republish to post on servers or to redistribute to lists, requires prior specific permission and/or a fee. Request permissions from permissions@acm.org.

MobiQuitous '18, November 5-7, 2018, New York, NY, USA

(c) 2018 Association for Computing Machinery.

ACM ISBN 978-1-4503-6093-7/18/11 .. \$15.00

https://doi.org/10.1145/3286978.3287012

\section{ACM Reference Format:}

Saiyma Sarmin, Nafisa Anzum, Kazi Hasan Zubaer, Farzana Rahman, and A. B. M. Alim Al Islam. 2018. Securing Highly-Sensitive Information in Smart Mobile Devices through Difficult-to-Mimic and Single-Time Usage Analytics. In EAI International Conference on Mobile and Ubiquitous Systems: Computing, Networking and Services (MobiQuitous '18), November 5-7, 2018, New York, NY, USA. ACM, New York, NY, USA, 10 pages. https://doi.org/10.1145/3286978. 3287012

\section{INTRODUCTION}

The use of smartphones has changed dramatically over the last decade. Their pervasive applications include sensitive usages, which are prone to face attackers anytime in different ways. Consequently, the issue of identifying valid users of these devices to ensure a high level of security has become a prime concern in recent times.

According to the study presented in [1], even though people are concerned about the security issues of smart devices, they are uncomfortable using available user identification techniques. The available user identification techniques can be divided into three basic categories [2], which are: (1) knowledge-based, (2) objectbased, and (3) biometric-based. Here, the first category typically includes passwords or PINs, which are often inconvenient for users as they require memorizing [3]. Furthermore, they can be stolen easily through eavesdropping or shoulder surfing [4]. The second category, i.e., object-based, techniques usually rely on possession of tokens. Here, if the tokens get lost or stolen, intruders will get access to unauthorized data. Though the third category or biometricbased techniques rely on the uniqueness of physical/behavioral characteristics of a person, they can be easily mimicked or stolen by an intruder nowadays [5].

Recently, few studies have been performed exploring user identification based on usage monitoring. One such study conducted in [6] proposes to continuously collect usage data and performs authentication accordingly [6-8]. Such techniques require passwords, fingerprints, or face detection continuously, which makes these techniques not only resource-hungry but also very user-unfriendly (requiring passwords or security questions) $[9,10]$ and costly (requiring extra devices for face-detection or fingerprint). Thus, to the best of our knowledge, a simple, single-time, easy-to-use, and a less resource-hungry user identification technique for highly-sensitive data is yet to be proposed in the literature.

In this paper, we address this issue by introducing a new singletime usage analytics based user identification technique. Taking into 
account two human behaviors - how the screen is being touched and how the device is being held, this technique exploits a new machine learning mechanism namely Mean-SD clustering. We perform experiments with several existing machine learning techniques in addition to our proposed technique to evaluate effectiveness of our considered human behaviors. We find that our proposed Mean-SD clustering technique exhibits considerable efficacy in user identification task exploiting the two behaviors while demanding a limited resource compared to other existing techniques. This happens as perhaps each user subconsciously carries a unique characteristics in terms of two behaviors, which is captured by our techniques. Further, our rigorous experimental evaluation confirms that mostly every user has a unique characteristics on touching the screen and holding the phone in combination, and consequently the user can be identified through these behavioral biometrics.

The strongest part of our proposed technique is that it exploits two behavioral metrics that are very difficult to mimic simultaneously. Therefore, it would be extremely hard for attackers to get access through breaching our proposed technique, which we confirm through getting zero false acceptance in our user evaluation.

Based on our work, we make the following set of contributions in this paper:

- We study single-time usage data collected from 33 participants. Our analysis through conventional clustering reveals that most of the participants are dominated by individual clusters and most of the clusters dominate only one participant. This finding paves a foundation for further investigation on usage data for the purpose of performing user identification based on the usage data.

- Next, we propose a novel light-weight machine learning technique called Mean-SD clustering for performing the user identification task.

- We confirm efficacy of our proposed clustering technique through identifying users from our data set of 33 participants with as low as 5\% False Acceptance Rate (FAR) and $6 \%$ False Rejection Rate (FRR). We perform necessary parameter tuning to achieve these false rates. The false rates in combination pertinent to user identification based on usage data are mostly lower compared to other available machine learning techniques that incur higher resource overhead.

- Later, we implement our proposed technique in three smart mobile devices and perform a user evaluation over 27 users with the devices. The user evaluation conducted under diversified situations and conditions demonstrates that the False Acceptance Rate remains at $0 \%$ even under attacks such as eavesdropping and shoulder surfing at an expense of a bit higher False Rejection Rate, a less benign aspect compared to False Acceptance Rate for sensitive data.

- Finally, we have collected data from 2 users continuously over 25 days in different operation states to evaluate the effectiveness of our technique in long-term usage in an uncontrolled environment. The user evaluation shows that the False Rejection Rate remains at as low as $1 \%$.

\section{RELATED WORK AND MOTIVATION}

Traditional smartphone based security systems include passwords, PINs, pattern locks and behavioral biometric techniques. All these techniques need memorizing except behavioral biometric techniques. Moreover, the secret contents of those techniques can be guessed or stolen as easily as shoulder surfing [11]. However, this system still needs memorizing as well as strongly depends on the keyboard layout of a specific language. Though biometric-based techniques, such as that exploiting fingerprints, cannot be guessed and give higher security but they can also be stolen or mimicked by imposters. Besides, they often require additional hardware, and hence, can be costly. One such approach studied in [12] introduces an authentication system based on simultaneous face and voice recognition. Because of using two biometric characteristics, this system is hard to break. However, it requires too much resource as well as cost, and therefore, does not suitable for mobile devices.

The study in [13] is another example of behavioral biometricbased system. It uses an additional identification layer immediately after the password/pattern approach. Furthermore, the study in [14] classifies users based on the usage of the user's hand movement while holding the device and the timing of touch-typing when the user enters a 4-digit PIN/password. Though both of these approaches give high security, they are still vulnerable as they use passwords/PIN. However, two other studies presented in [15] and [16] also develop authentication systems, which enhance the level of security. Here, the study in [15] implements biometric analysis in combination with pattern recognition and [16] uses multi-touch gestures. However, these mechanisms present difficulties to users as it demands complex gestures. Moreover, studies conducted on run-time authentication $[6,17,18]$ continuously perform user identification in the background. Even though these systems give higher level of security, these are not suitable for deploying in low-resource devices as they consume more power and exhibit to be extremely resource-hungry. Besides, the study in [19] is an example of behavioral biometric-based system. This system uses mobile device touchscreens and their built-in inertial sensors and vibration motor to detect hand posture and pressure on commodity phones, rather than to identify valid users.

In summary, conventional single-time user identification systems exhibit a threat of being stolen or mimicked. Consequently, the initial motivation for our research arose from the need to provide a difficult-to-mimic user identification technique for mobile devices. Although continuous identification systems give more security, their strength depends on run-time data capturing and rigorousness of continuous data analyzing, which demand substantial CPU and memory making them unsuitable for low-resource devices. Therefore, the motivation of our work evolve on developing a single-time user identification technique that would require tracking of user's usage only for one time. We choose to explore the use of user's touch and holding orientation based usage monitoring for the identification since they usually have the benefits of not involving additional physical gadgets and not demanding the user's special attention for a long period of time. Here, the touch-based usage monitoring is free from the hassle of memorization. Alongside, another easily-capturable usage metric is holding orientation 
of a smartphone, which exhibits a good potential to vary from userto-user in a subtle way. To the best of our knowledge, combining the touch-based usage and holding orientations are yet to be investigated in the literature. Therefore, in this paper, we attempt to perform the investigation for user identification.

\section{PROPOSED TECHNIQUE FOR USER IDENTIFICATION}

In this section, we describe our proposed user identification technique in details. Our working methodology involves two phases: training phase and user identification phase. Two phases are elaborated in this section. Here, first, we present the overview of working methodology along with the steps in the training phase. Subsequently, we elaborate the user identification technique in details.

\subsection{Overview of Underlying Methodology}

Our proposed technique requires training data, which is the core of the identification technique. The training data is collected from the user and immediately used to train the application. The training results are stored in the device, which will be used to perform the identification task. The training data will no longer need to be collected unless needed for any specialized reasons such as significant change in usage for experiencing an accident or so. After training, the system will be ready to identify its valid user. Identification starts by capturing usage data provided by the person who is using the device currently. This usage data is then compared against the stored training data. Based on the result of the comparison, user identification is performed.

\subsection{Training Phase}

Training phase includes two steps-collection training data and generating training results. This training phase occurs only once at the first time of starting the application when the data is given by the owner of the device. Later the device automatically identifies its owner based on the result obtained from this training phase. Two steps of the training phase are elaborated in the next subsections.

3.2.1 Collecting Training Data. Before designing our proposed technique, we observe and study user behavior of several acts based on their touch usages. As a result of the study, we identified that we can extract 13 features from any user's touch usage. These 13 features individually do not show any significant characteristic that can specify a user. However, in combination, these features contribute to the unique identification of a user. These features are six touch coordinates (three coordinates each at start and end positions of a swipe), finger pressures (the force applied) over each of the start and end finger positions, velocity during the swipe (X-axis and Y-axis), hold-time (duration of the interval between starting and ending of the swipe), tilt angle, and rotation matrix while pressing a button.

3.2.2 Training over the Collected Data. As discussed before, each usage data contains 13 features. Therefore, each usage data can be considered as a 13-dimensional vector. In the data collection phase, we collect such data sets from the user for training. Consequently, we can say that in 13-dimensional space, data collected from each user forms separate clusters. Our proposed solution identifies the cluster of each user by two different pieces of information - cluster centroid, which is the mean of all cluster members, and cluster size, which is calculated by our own-developed heuristic-based equation. In the training phase, after collecting training data, we calculate both the cluster information and store them in the device.

The cluster size $(C)$ is varied and determined by taking a weighted sum of the average and standard deviation, which can be expressed by Eq. 1 presented as follows:

$$
C=\left(M_{\mathrm{avg}} \times W_{\mathrm{avg}}\right)+\left(S_{\mathrm{sd}} \times W_{\mathrm{sd}}\right)
$$

where $M_{\text {avg }}$ represents average and $S_{\text {sd }}$ represents the standard deviation over all the cluster members of a cluster in training data. Besides, $\mathrm{W}_{\text {avg }}$ and $\mathrm{W}_{\text {sd }}$ are the weights of average and standard deviation respectively.

\subsection{User Identification Process}

After the training phase is completed, user identification starts by collecting a single usage data of 13 features from the current user. This data will indicate a point in the 13-dimensional region, which will be used to identify the user. From the training phase, we already have the cluster centroid and the cluster size stored in the device, which represents the usage biometric characteristics of a user. Now, the distance of the new point from the cluster centroid is calculated. The user is identified as the valid user if the new point falls within the cluster size. Otherwise, the user will be identified as an invalid user. Our proposed clustering technique identifies users based on their own clusters, which are defined by the centroids and sizes of their clusters. The cluster size can be varied by turning weights of mean and standard deviation of the cluster members. For this reason, we name our proposed technique as Mean-SD clustering.

\subsection{Performance Measures}

In the training phase, we use training data sets to calculate the cluster parameters, i.e., cluster centroids and cluster sizes. For the purpose of performance evaluation of our proposed technique, we collect training data sets from different users. Therefore, we get multiple clusters where each cluster indicates a specific user. Similarly, we collect test data sets in order to evaluate efficacy and effectiveness of the training. The performance measures in our evaluation are False Acceptance Rate (FAR) and False Rejection Rate (FRR). The FAR indicates at what percentage the system inaccurately accepts an invalid user as a valid one. FRR indicates at what percentage the system incorrectly rejects a valid user. These two measures give probability indicating how efficiently the system will perform.

\section{EXPERIMENTAL DESIGN FOR PERFORMANCE EVALUATION OF OUR PROPOSED TECHNIQUE}

We perform a set of experiments in order to evaluate the performance and accuracy of our proposed technique. Our experiments include data collection, analysis, implementing our proposed solution, and evaluating the results. In this section, we present details on the experimental setup and demography of collected data. 


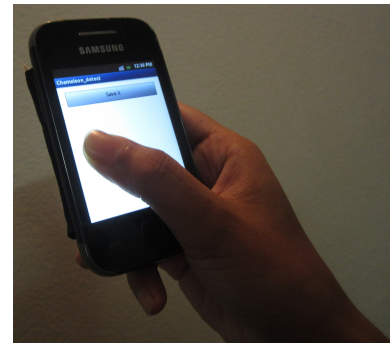

(a) Task 1: Fixed swipe over the touchscreen

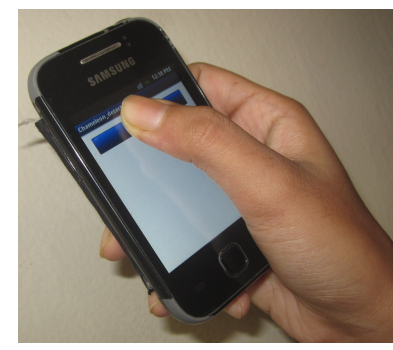

(b) Task 2: Press a specific button over the touchscreen
Figure 1: Data collection procedure in our experimentation

\subsection{Data Collection Platform}

At first, we collect user's touch-based usage data in order to perform the experiments. Data is to be collected from a device having several sensors that can collect usage data and a mechanism that can store the collected data. For this reason, we develop an Android application that captures and stores our intended usage data of a user. We use a Samsung Galaxy Young gt-s5360A [20] device for this purpose. The device possesses 384MB RAM of memory, 832 $\mathrm{MHz}$ ARMv6 processor, and a Broadcom VideoCore IV.

\subsection{Application for Data Collection}

As discussed in Section 3, our data collection application records usage patterns of different users from the perspective of all the 13 features. We develop the application in Android 2.3 (Gingerbread). The application asks a user to do two simple tasks - a fixed swipe on the touchscreen and then press a specific button. Here, start and end positions along with trajectory of the fixed swipe are defined solely by the user. We term the swiping task as fixed swipe, as it remains fixed for the user. How the user interacts with the touchscreen during swiping completely depends on the user's choice. In Fig. 1, we present a user in action while using the application. Following the same process, we collect data from several users. Demography of all the users is presented in the next section.

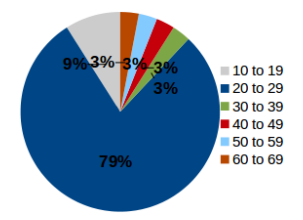

(a) Age range (years)

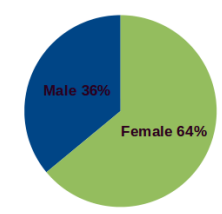

(b) Gender

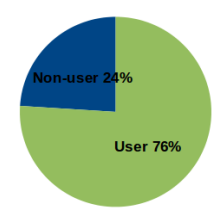

(c) Touchscreen usage
Figure 2: Demography of participating users

\subsection{Demography of the Users}

We collect data from 33 participants for our experiment. The participants cover different age groups (from 11 to 67 years old), different genders, and different levels of experience in interacting with touchscreen electronic devices. We show the demography of participants in Fig. 2. Here, Fig. 2a shows that our participants mostly exhibit youth having an age range of 20 to 30 years. We have picked such skewed diversity in terms of age range, as survey studies existing in the literature [21], [22] exhibit a similar skewed diversity in favor of youth for usage of touchscreen-based electronic devices. Besides, Fig. 2b presents that we have covered a significant number of both male and female in our experiment. Finally, Fig. 2c shows that our experiment covers non-users of touchscreen-based electronic devices in accordance with users of touchscreen electronic devices. We collect data from all of the participating users for 15 times. Among them, we keep 10 sets of data for training and 5 sets of data for testing. We perform several iterations of the same process adopting the same number (10 for training, 5 for testing) of data sets, however, drawn in a random manner from the available 15 sets of data.

\section{EXPERIMENTAL DATA ANALYSIS}

At the beginning of our study, we conduct several experiments in order to understand the behavior of the collected data. At first, we perform unsupervised clustering and investigate characteristics of generated clusters to analyze whether there lies any user-cluster mapping as per the extracted features. After that, we conduct a thorough analysis and investigate if we can assign a user to a specific cluster. This way we can identify a user as the original one in case we get usage data within the cluster and identify other users as aliens in case we get usage data outside the cluster. Furthermore, in the following subsections, we present our analytical outcomes along with how far we could achieve the objectives.

\subsection{Outcomes of Unsupervised Clustering}

We attempt to perform unsupervised clustering over the training data collected without providing any information about the owner of the data. Thus, it creates clusters of related data, where each member of a cluster exhibits different characteristics from members of another cluster. Here, we find 26 clusters. Note that, the number of clusters is close to the number of participants, i.e. 33. Table 1 shows the results of this unsupervised clustering. Here, each column of the table represents a user and each row denotes a cluster. After analyzing the data presented in Table 1, we can find that most of the rows in the table, i.e., clusters obtained by unsupervised clustering, is covered by a few columns, i.e., by a few of the participants. Besides, most of the participants cover only a few clusters, as most of the columns cover only a few rows. In the table, both the blue and red shaded cells exhibit the covering. A bit more investigation reveals that most of the clusters are dominated by a very small number of users, and it is very close to 1 in most of the cases. Besides, most of the users dominate only a very small number of clusters, which is again very close to 1 in most of the cases. Red shaded cells in the table represent the dominances. From the above analysis, we can say that there might be a near one-toone mapping between the users and the clusters. Consequently, it might be possible to efficiently cluster the data such that from the cluster information, the users could be identified. We attempt to do so in the next subsection. 


\begin{tabular}{|c|c|c|c|c|c|c|c|c|c|c|c|c|c|c|c|c|c|c|c|c|c|c|c|c|c|c|c|c|c|c|c|c|c|}
\hline $\begin{array}{c}\text { User } \\
\text { Cluster }\end{array}$ & 1 & 2 & 3 & 4 & 5 & 6 & 7 & 8 & 9 & 10 & 11 & 12 & 13 & 14 & 15 & 16 & 17 & 18 & 19 & 20 & 21 & 22 & 23 & 24 & 25 & 26 & 27 & 28 & 29 & 30 & 31 & 32 & 33 \\
\hline 1 & 2 & 0 & 0 & 0 & 0 & 0 & 0 & 0 & 1 & 0 & 0 & 0 & 0 & 0 & 0 & 2 & 0 & 0 & 0 & 1 & 0 & 0 & 0 & 0 & 0 & 0 & 0 & 0 & 0 & 0 & 0 & 0 & 0 \\
\hline 2 & 4 & 4 & 5 & 7 & 0 & 6 & 0 & 4 & 0 & 0 & 2 & 0 & 5 & 0 & 0 & 0 & 6 & 3 & 0 & 0 & 0 & 2 & 0 & 0 & 0 & 0 & 0 & 2 & 0 & 0 & 0 & 0 & 2 \\
\hline 3 & 0 & 0 & 0 & 0 & 4 & 0 & 0 & 0 & 0 & 0 & 0 & 0 & 0 & 0 & 0 & 0 & 0 & 0 & 0 & 0 & 0 & 0 & 0 & 0 & 0 & 0 & 0 & 0 & 0 & 0 & 0 & 0 & 0 \\
\hline 4 & 1 & 0 & 0 & 0 & 0 & 0 & 0 & 0 & 4 & 1 & 0 & 0 & 0 & 0 & 0 & 3 & 0 & 0 & 0 & 0 & 0 & 0 & 0 & 0 & 0 & 0 & 0 & 0 & 0 & 0 & 0 & 0 & 0 \\
\hline 5 & 0 & 0 & 0 & 0 & 0 & 0 & 0 & 0 & 1 & 0 & 0 & 0 & 0 & 0 & 0 & 0 & 0 & 0 & 0 & 0 & 0 & 0 & 0 & 0 & 0 & 0 & 0 & 0 & 0 & 0 & 0 & 0 & 0 \\
\hline 6 & 0 & 0 & 0 & 0 & 0 & 0 & 0 & 0 & 0 & 0 & 1 & 0 & 0 & 0 & 0 & 0 & 0 & 0 & 0 & 0 & 0 & 0 & 0 & 0 & 0 & 0 & 0 & 0 & 0 & 0 & 0 & 0 & 0 \\
\hline 7 & 0 & 0 & 0 & 0 & 0 & 0 & 0 & \begin{tabular}{l|l} 
\\
\end{tabular} & 0 & 0 & 0 & 1 & 0 & 0 & 0 & 0 & 0 & 0 & 0 & 0 & 0 & 0 & 0 & 0 & 0 & 0 & 0 & 0 & 0 & 0 & 0 & 0 & 0 \\
\hline 8 & 0 & 1 & 0 & 0 & 0 & 1 & 0 & 0 & 0 & 0 & 0 & 0 & 1 & 4 & 0 & 0 & 0 & 4 & 0 & 0 & 0 & 0 & 0 & 0 & 0 & 0 & 0 & 0 & 0 & 0 & 0 & 0 & 0 \\
\hline 9 & 0 & 0 & 0 & 0 & 2 & 0 & 0 & 0 & 0 & 0 & 0 & 1 & 0 & 0 & 6 & 0 & 0 & 0 & 0 & 0 & 0 & 1 & 2 & 0 & 0 & 0 & 0 & 0 & 0 & 0 & 0 & 0 & 0 \\
\hline 10 & 0 & 0 & 0 & 0 & 0 & 0 & 0 & \begin{tabular}{l|l} 
\\
\end{tabular} & 0 & 0 & 0 & 0 & 0 & 0 & 0 & 1 & 0 & 0 & 0 & 0 & 0 & 0 & 0 & 0 & 0 & 0 & 0 & 0 & 0 & 0 & 0 & 0 & 0 \\
\hline 11 & 0 & 0 & 0 & 0 & \begin{tabular}{l|l} 
\\
\end{tabular} & 0 & 0 & 0 & 0 & 1 & 0 & 0 & 0 & 0 & 0 & 1 & 0 & 0 & 0 & 0 & 1 & 0 & 0 & 0 & 0 & 0 & 0 & 0 & 0 & 0 & 0 & 0 & 0 \\
\hline 12 & 0 & 0 & 1 & 0 & 0 & 0 & 5 & 1 & 0 & 0 & 4 & 4 & 0 & 0 & 0 & 0 & 0 & 0 & 7 & 0 & 0 & 1 & 3 & 4 & 0 & 0 & 0 & 1 & 0 & 0 & 0 & 0 & 0 \\
\hline 13 & 0 & 0 & 0 & 0 & 1 & 0 & 0 & 0 & 0 & 1 & 0 & 0 & 1 & 0 & 0 & 0 & 0 & 0 & 0 & 6 & 0 & 0 & 1 & 0 & 0 & 0 & 0 & 0 & 0 & 0 & 0 & 0 & 0 \\
\hline 14 & 0 & 0 & 0 & 0 & 0 & 0 & 0 & 0 & 1 & 4 & 0 & 0 & 0 & 0 & 0 & 0 & 0 & 0 & 0 & 0 & 5 & 0 & 0 & 0 & 0 & 0 & 0 & 0 & 0 & 0 & 0 & 0 & 0 \\
\hline 15 & 0 & 0 & 1 & 0 & 0 & 0 & 2 & 2 & 0 & 0 & 0 & 1 & 0 & 0 & 1 & 0 & 0 & 0 & 0 & 0 & 1 & 3 & 1 & 0 & 0 & 0 & 0 & 0 & 0 & 0 & 0 & 0 & 0 \\
\hline 16 & 0 & 0 & 0 & 0 & 0 & 0 & 0 & 0 & 0 & 0 & 0 & 0 & 0 & 0 & 0 & 0 & 0 & 0 & 0 & 0 & 0 & 0 & 0 & 1 & 0 & 0 & 0 & 0 & 0 & 0 & 0 & 1 & 0 \\
\hline 17 & 0 & 0 & 0 & 0 & 0 & 0 & 0 & 0 & 0 & 0 & 0 & 0 & 0 & 0 & 0 & 0 & 0 & 0 & 0 & 0 & 0 & 0 & 0 & 0 & 3 & 0 & 0 & 1 & 0 & 3 & 2 & 0 & 0 \\
\hline 18 & 0 & 0 & 0 & 0 & 0 & 0 & 0 & 0 & 0 & 0 & 0 & 0 & 0 & 0 & 0 & 0 & 0 & 0 & 0 & 0 & 0 & 0 & 0 & 0 & 0 & 1 & 0 & 0 & 0 & 0 & 0 & 0 & 0 \\
\hline 19 & 0 & 0 & 0 & 0 & 0 & 0 & 0 & 0 & 0 & 0 & 0 & 0 & 0 & 0 & 0 & 0 & 0 & 0 & 0 & 0 & 0 & 0 & 0 & 0 & 0 & 3 & 1 & 0 & 0 & 2 & 0 & 1 & 0 \\
\hline 20 & 0 & 0 & 0 & 0 & 0 & 0 & 0 & \begin{tabular}{l|l} 
\\
\end{tabular} & 0 & 0 & 0 & 0 & 0 & 0 & 0 & 0 & 0 & 0 & 0 & 0 & 0 & 0 & 0 & 0 & 0 & 0 & 1 & 1 & 0 & 0 & 0 & 0 & 0 \\
\hline 21 & 0 & 0 & 0 & 0 & 0 & 0 & 0 & 0 & 0 & 0 & 0 & 0 & 0 & 0 & 0 & 0 & 0 & 0 & 0 & 0 & 0 & 0 & 0 & 0 & 0 & 0 & 5 & 0 & 7 & 0 & 0 & 0 & 0 \\
\hline 22 & 0 & 0 & 0 & 0 & 0 & 0 & 0 & 0 & 0 & 0 & 0 & 0 & 0 & 0 & 0 & 0 & 0 & 0 & 0 & 0 & 0 & 0 & 0 & 0 & 0 & 0 & 0 & 0 & 0 & 0 & 1 & 0 & 0 \\
\hline 23 & 0 & 0 & 0 & 0 & \begin{tabular}{l|l} 
\\
\end{tabular} & 0 & 0 & \begin{tabular}{l|l} 
\\
\end{tabular} & 0 & 0 & 0 & 0 & 0 & 0 & 0 & 0 & 0 & 0 & 0 & 0 & 0 & 0 & 0 & 0 & 0 & 1 & 0 & 0 & 0 & 0 & 0 & 0 & 0 \\
\hline 24 & 0 & 0 & 0 & 0 & 0 & 0 & 0 & 0 & 0 & 0 & 0 & 0 & 0 & 0 & 0 & 0 & 0 & 0 & 0 & 0 & 0 & 0 & 0 & 0 & 0 & 0 & 0 & 0 & 0 & 0 & 0 & 1 & 0 \\
\hline 25 & 0 & 0 & 0 & 0 & 0 & 0 & 0 & 0 & 0 & 0 & 0 & 0 & 0 & 0 & 0 & 0 & 0 & 0 & 0 & 0 & 0 & 0 & 0 & 0 & 1 & 0 & 0 & 2 & 0 & 0 & 0 & 3 & 0 \\
\hline 26 & 0 & 2 & 0 & 0 & 0 & 0 & 0 & 0 & 0 & 0 & 0 & 0 & 0 & 3 & 0 & 0 & 1 & 0 & 0 & 0 & 0 & 0 & 0 & 2 & 3 & 2 & 0 & 0 & 0 & 2 & 4 & 1 & 5 \\
\hline
\end{tabular}

Table 1: Findings of unsupervised clustering of swipe patterns of the users (each row represents a cluster and each column represents a user; most of the clusters are dominated by only one user, which is represented in red cells; most of the users dominate only one cluster, which is demonstrated in blue cells)

\subsection{User Identification using Mean-SD Clustering Technique}

To perform an in-depth investigation of our proposed Mean-SD clustering over our collected data, we cluster the collected data from the perspective of both individual single feature and multiple features. We perform a number of iterations with such clustering tasks over randomly chosen different sets of data for both individual features and all features. We perform these clustering tasks to lead towards efficient user identification. Moreover, we investigate the effect of allowing mismatch among the features in our identification process. We describe all the identification processes below.

5.2.1 Identification Based on Single Feature. At first, we evaluate the performance of our proposed technique based on every single feature. According to Eq. 1, the size of a cluster depends on two parameters - weight of standard deviation and weight of average. Here, we analyze the efficacy of identification exploiting single feature by varying the cluster size through changing only one parameter while keeping the other one fixed. We further investigate the consequences of changing weights of average and standard deviation by analyzing their impacts on FAR and FRR. We present the individual impacts in Fig. 3. Here, Fig. 3a demonstrates the effect of changing the weights on FAR for a single feature which is rotation about the $\mathrm{Z}$-axis while pressing the button in our developed application. This figure demonstrates that the FAR increases rapidly with an increase in the cluster size by increasing the weights. Note that the cluster size can be increased through increasing either of the weights individually or both the weights simultaneously. Similarly, Fig. $3 \mathrm{~b}$ presents the effect of changing the weights on FRR for a single feature. However, in contrast to the previous case, in this case, the FRR decreases with an increase in the cluster size. After analyzing the impacts of different possible values of the weights, we can find that the results obtained with the feature of rotation about the Z-axis while pressing the button having a cluster size of $(A V G \times 5 \%+S D \times 370 \%)$ demonstrates the best possible accuracy where the combination of lowest values of FAR are $11 \%$ and $8 \%$ accordingly.

5.2.2 Identification Based on Multiple Features. Following the same process, we compute FARs and FRRs for all of the features. Note that, even though FAR and FRR vary in different ways for different features, both FAR and FRR exhibit substantial values. Thus, FAR and FRR for only one feature exhibit low accuracy (up to $80 \%$ at best) rendering it is not a feasible solution. Now, after reaching this extent, we apply the clustering technique to a different combinations of features. To do so, we attempt for the same identification task based on matching all features together. The purpose behind such attempt is two-folded - firstly, to improve the accuracy of identification, and secondly, to make the system more secure as it is comparatively much more difficult for a malicious user to simultaneously mimic more than one usage behavior of the original user.

In our analysis based on matching multiple features, we utilize the same weight of average for all the features as well as the same weight of standard deviation for all the features. Here, we also independently vary both the weights. We present the effect of changing the weights of average and standard deviation for all features on FAR in Fig. 4a. Here, the finding is similar to that we have already found for a single feature. The FAR increases with an increase in the cluster size. Similarly, the FRR decreases with an 


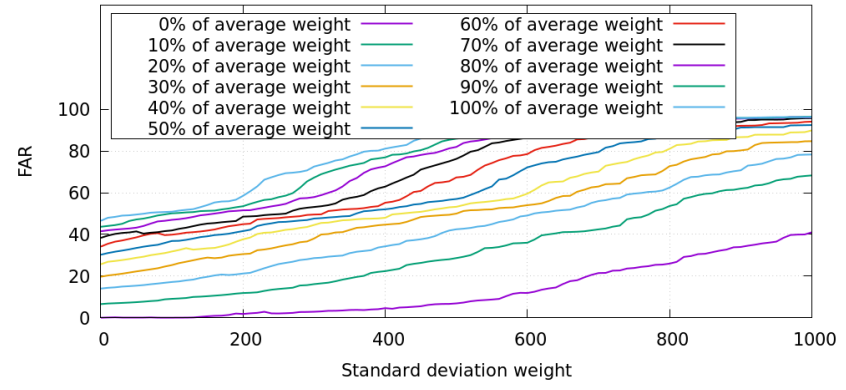

(a) Effect of change in the weight of average and the weight of standard deviation on FAR

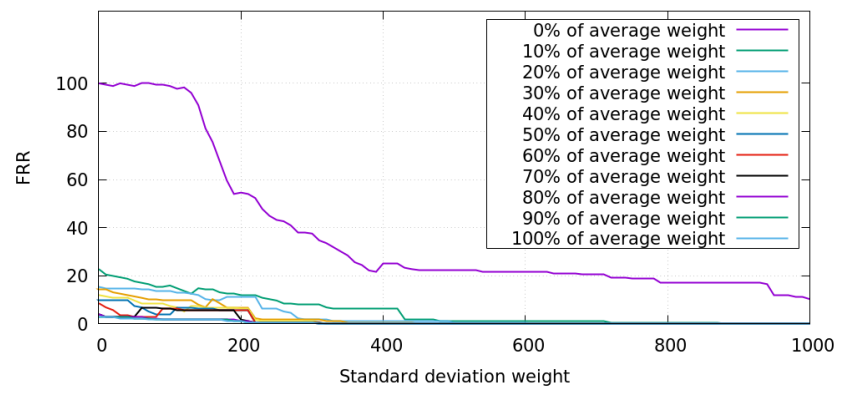

(b) Effect of change in the weight of average and the weight standard deviation on FRR

Figure 3: Effect of change in the weight of average and the weight of standard deviation on FAR and FRR for the feature providing best-possible outcomes, which is rotation about the $\mathrm{Z}$-axis while pressing the button in our application

increase in the cluster size. Fig. 4b shows this result for FRR. The best result obtained while considering all features in combination is for the cluster size of $(A V G \times 5 \%+S D \times 530 \%)$. Fig. $4 \mathrm{c}$ presents variations in FAR and FRR pertinent for this cluster size. Here, the best possible outcome provides only $5 \%$ FAR and $6 \%$ FRR.

Now, if we compare the results pertinent to considering only a single feature and the results pertinent to considering all features in combination, we can find some interesting observations. After analyzing Fig. 3a and Fig. 4a, we find that the FAR decreases if we consider all features in combination compared to the case of considering only a single feature. In the case of FRR, the scenario gets changed. Here, FRR decreases if we consider only a single feature compared to the case of considering all features in combination. Fig. $3 \mathrm{~b}$ and Fig. $4 \mathrm{~b}$ present such a case of getting decreased FRR through considering only a single feature.

Even though we get two opposing trends in FAR and FRR while considering the features in isolation and while considering all the features in combination, we can achieve the best possible outcome in the case of considering all features in combination. Fig. $4 \mathrm{c}$ validates the phenomena of achieving the better outcome through considering all the features in combination.

5.2.3 Allowing Mismatch over the Features. Another important investigation we perform is related to allowing mismatch in features

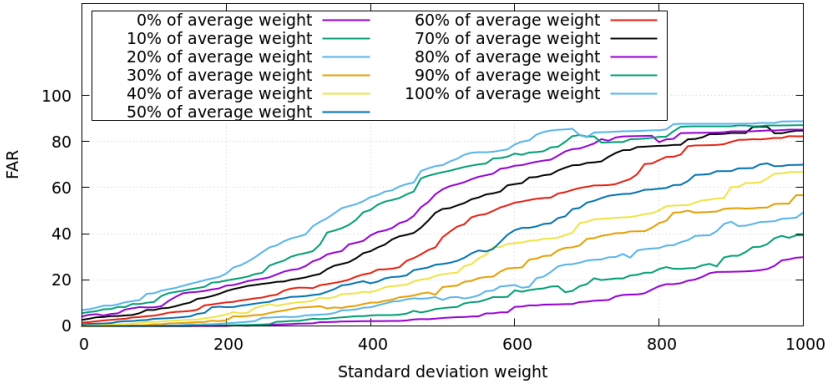

(a) Effect of change in the weight of average and the weight of standard deviation on FAR

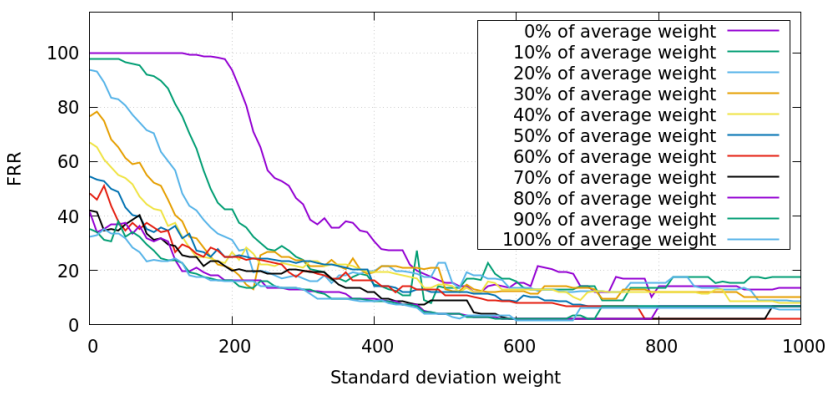

(b) Effect of change in the weight of average and the weight of standard deviation on FRR

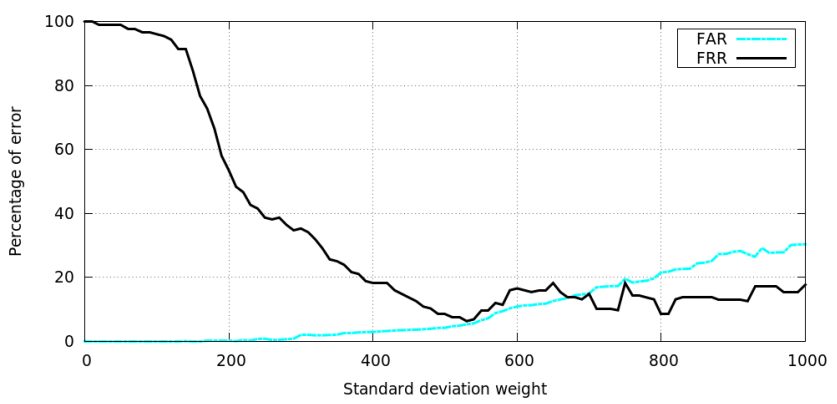

(c) FAR and FRR while considering all the features providing the best-possible outcome

Figure 4: Effect of changing the weights of average, standard deviation, and allowing mismatch on FAR and FRR while considering all the features

on the best possible case while considering all features. Here, we allow mismatching for different numbers of features while performing the user identification task. We find that the accuracy decreases while allowing mismatch over features. With an increase in the number of allowed mismatches, the FAR increases up to $100 \%$ and the FRR decreases up to $0 \%$. Thus, allowing mismatches over the features increases FAR more than it decreases FRR, and thus ultimately undermines the overall accuracy. 


\begin{tabular}{|c|c|c|c|c|}
\hline Technique & Parameter & FAR & FRR & Run-time $(\mu \mathrm{s})$ \\
\hline \multirow{3}{*}{$\mathrm{k}-\mathrm{NN}[23]$} & $\mathrm{k}=1$ & $1 \%$ & $9 \%$ & 110 \\
\cline { 2 - 5 } & $\mathrm{k}=3$ & $1 \%$ & $10 \%$ & 150 \\
\cline { 2 - 5 } & $\mathrm{k}=5$ & $0 \%$ & $11 \%$ & 160 \\
\hline Decision tree [24] & - & $0 \%$ & $16 \%$ & 1.94 \\
\hline Kessler [25] & - & $2 \%$ & $41 \%$ & 2.04 \\
\hline Mean-SD & - & $5 \%$ & $6 \%$ & 1.6 \\
\hline
\end{tabular}

Table 2: Comparative analysis of classical machine learning algorithms and our proposed technique

\section{COMPARATIVE ANALYSIS OF CLASSICAL MACHINE LEARNING ALGORITHMS WITH OUR PROPOSED MEAN-SD CLUSTERING}

We analyze our collected usage data with several classical machine learning algorithms to perform a comparative evaluation of the performance of our proposed solution against the performances of classical machine learning algorithms.

We choose three classical machine learning algorithms - kNearest Neighbor (k-NN) [23], Decision tree [24], and a multiclass perceptron algorithm namely Kessler's construction [25] considering their wide acceptability in the literature. We present the findings of these machine learning algorithms in Table 2. We have got these findings through performing our analysis on a machine having a Core i5 processor of $2.5 \mathrm{GHz}$ and 4 GB memory.

Our experiments show that the k-NN algorithm provides lower false rates compared to Decision tree and Kessler's construction algorithms. More specifically, the FRR is significantly higher for Decision tree and Kessler's construction compared to k-NN. Here, the FRR gets decreased for $\mathrm{k}-\mathrm{NN}$ with a decrease in the value of $\mathrm{k}$. However, with the decrease in the value of $k$, the FAR gets increased.

Table 2 also presents the running time needed to identify a user using the machine learning algorithms and our proposed Mean-SD clustering technique. We can see that $\mathrm{k}-\mathrm{NN}$ needs more execution time than other approaches. This is because, for each test sample, $\mathrm{k}-\mathrm{NN}$ checks against all the trained samples and finds the best $\mathrm{k}$ matches. Thus, the time for k-NN algorithm increases with an increase in the number of samples in the train data-set. Therefore, k-NN consumes a huge amount of memory as it stores the whole training data-set. Therefore, though $\mathrm{k}-\mathrm{NN}$ has lower FAR and FRR in combination ( $1 \%$ less than our case), the increased memory usage and longer execution time make it unsuitable for practical implementation. Decision tree algorithm also needs a considerable memory to store the whole tree information. Moreover, the FRR is too high in this case. Nonetheless, Kessler's construction has the highest FRR among all these approaches. Comparing performances of these classical machine learning algorithms with that of our proposed Mean-SD clustering technique, we find that our proposed technique provides the best result with 5\% FAR and 6\% FRR along with the lowest execution time and memory usage.

\section{EXPERIMENTAL FINDINGS}

This section describes the findings of our experiment. According to our experimental outcomes, we get the following findings:
- Our experimental results over several machine learning algorithms demonstrate that our proposed Mean-SD clustering achieves nearly the best accuracy of $89 \%$ (only k-NN with $\mathrm{k}=1$ can have $90 \%$ accuracy, higher than this). However, our proposed technique demand significantly less memory and less computation time compared to the other algorithms making it suitable for pragmatic application.

- The experimental results of our proposed Mean-SD clustering demonstrate that the FAR increases rapidly with an increase in the cluster size and the FRR decreases rapidly with an increase in the cluster size. These results are shown in Fig. $3 \mathrm{a}$ and Fig. 3b. Here, the best possible accuracy with a single feature is obtained with rotation about the $\mathrm{Z}$-axis while pressing the button having the cluster size of $(A V G \times 0.05+$ $S D \times 3.7)$ which experiences $11 \%$ FAR and $8 \%$ FRR.

- Our experiment discovers that relying on single feature is comparatively less secure as it is easy to reproduce a single feature by others contributing the FAR as well as usage data may vary time to time for an individual user contributing to FRR.

- Considering all features in combination provides better accuracy as shown in Fig. 4c. We achieve the best result while considering all features in combination with the cluster size of $(A V G \times 0.05+S D \times 5.3)$. Here, the best possible outcome provides only $5 \%$ FAR and $6 \%$ FRR.

- Accuracy decreases while allowing mismatch over features $(0-12)$.

\section{USER EVALUATION}

To evaluate the actual performance of our proposed technique, we develop our Mean-SD clustering algorithm in an Android application. Here, all the calculations needed in our technique are done in the device, and we did not use any library or any cloud service. For a specific user, the application initially takes a training dataset from the user to train the device so that it can identify the user, and thus, distinguish attackers. To perform the identification task, we set the values of $\mathrm{W}_{\text {avg }}$ and $\mathrm{W}_{\text {sd }}$ as 0.05 and 5.3 following the weights pertinent to the best case of our earlier results.

\subsection{User Demography}

We let 24 untrained users use a system having our developed application. All of the users are from the age range $20-29$ years. Alongside, 15 users are male and 9 users are female. Besides them, we let another three participants to use the application for 2-3 days so that they become used to it. All of them were male from an age range of $20-25$ years. We refer them as trained users.

\subsection{Device Specifications}

To analyze the performance of our application with the presence of a different number of sensors having different capabilities, we use the following three devices in our user evaluation:

Samsung GALAXY Tab 10.1 LTE SC-01D [26]: This device has Android version 3.2, dual-core $1.5 \mathrm{GHz}$ CPU, and 1 GB RAM. This device contains sensors for touch detection, pressure detection, finger movement velocity detection, and tilt angle detection. HTC Desire 626 [27]: This device has Android version 4.4.4, quad- 
core 1.2 GHz CPU, and 1 GB RAM. This device contains sensors for touch detection and finger movement velocity detection only. This device was used for long-term evaluation.

Asus Zenfone 2 Laser [28]: This device has Android version 5.0, quad-core 1.2 GHz Cortex-A53 CPU, and 2 GB RAM. This device contains sensors for touch detection and finger movement velocity detection only. This device was also used for long-term evaluation.

\subsection{Experimental Settings}

In order to determine the minimum number of training data needed to train the application, we let our three trained users to train the device with a different number of training data and measure corresponding FRR. We can see the outcome of this experiment in Fig. 5a that the FRR mostly saturates to a small value after 9-10 training data. From this observation, we set the standard number of training data required to train the application to 10 .

After that, we let all our 24 untrained users to use the application both on the tablet and on the phone. First, we collect 10 training data from each of the users for each of the devices. Then they performed their test swipes with a goal to be identified in a legitimate manner The number of legitimate test swipes per user varied from five to ten. In total, there were 158 test swipes for the phone and 199 test swipes for the tablet device. Here, in 57 times among the swipes, intruders attempted to mimic true users after shoulder surfing with a guided malicious intent.

We also let our three trained users to use the app on the tablet device in different states - standing, sitting, walking, and jogging. Here, in the training phase, they provided training swipe patterns while sitting on a chair, and later, they swiped in the different states while attempting to be identified in the testing phase.

\subsection{Findings From User Evaluation}

From evaluation with 24 users, the tablet device shows extremely low FAR of absolute $0 \%$. On the other hand, the mobile device shows a bit higher FAR of 7\%. On the contrary, the mobile device shows a lower FRR (15\%) than that of the tablet device (39\%). In case of the tablet device, the FAR retains the value $0 \%$ even for making attempts through shoulder surfing. This ensures that the solution has a very high precision in blocking intruders, which is the prime motivation for this research. To perform the identification task on a test swipe, the application takes only $45.6 \mathrm{~ms}$ on the tablet and only $3.9 \mathrm{~ms}$ on the phone on an average (taken from an average over ten samples). The application uses 30-33\% of CPU with $26 \mathrm{MB}$ memory on the tablet and $25-30 \%$ of CPU with $37 \mathrm{MB}$ memory on the phone during the small period of identification. Besides, while being only active in idle manner performing no identification task, the application takes only $0.1 \%$ CPU or even less. These measurements demonstrate a guarantee of our claim on the proposed technique of being very lightweight. Note that the time required for single identification task differs from the value mentioned in Table 2, as values presented in the table were for isolated data analysis using a laptop whereas the values reported in this section are from our implementation on the tablet and phone. Besides, among our adopted two devices, the mobile device has fewer sensors than the tablet device. Therefore, the two devices exhibit different performances. With a higher number of available

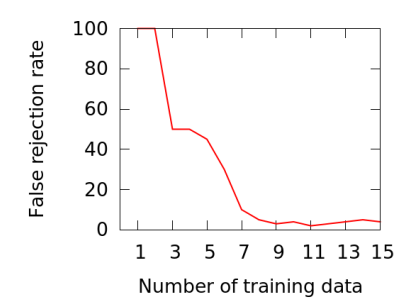

(a) Performance in terms of FRR with a change in the number of training data

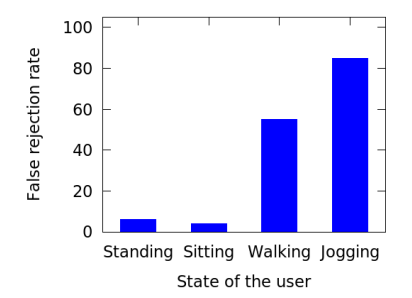

(c) Performance in terms of FRR for varying states of a user

\section{Figure 5: Performance measure of our proposed Mean-SD} clustering technique in real implementation

sensors, the tablet provides better accuracy in identifying intruders. Nonetheless, owing to the usage of more sensors, the tablet also exhibits more rejections to legitimate users.

8.4.1 Performance Enhancement with Increasing Usage. To further analyze the performance of our application, we let three untrained users to continuously use the application and record success rates with the number of attempts as shown in Fig. 5b. Analyzing the figure, we can find that though the success rate fluctuates and remains low initially, it gets better over the time ( $80 \%$ after 30 attempts) when the user becomes more used to the application.

8.4.2 Accuracy in Different States of A User. We have measured the accuracy when a trained user attempts to get identified through our system in different states such as standing, sitting, walking, and jogging. We find that the FRR for standing and sitting states are 4-6\%. However, for the moving states such as walking and jogging, the FRR is $55-85 \%$ indicating that our proposed technique needs to be used mostly in a stationary state to get entered into a device. We present the results in Fig. 5c.

8.4.3 Accuracy with Long-time Usage. To further analyze the performance of our application, we let two of our untrained users to continuously use the application and record success rates in different operational states such as sitting, walking, lying; in different mental and physical states such as depressed, happy, exhaustive, and neutral; and in different holding states such as left-handed or right-handed. They used this application on Asus Zenfone 2 Laser [28] and HTC Desire 626 [27] over consecutive 25 days. Here, we find that, though the success rate is too high for User1, the false 
rejection rate remains high for User2. After completion of this study, we took opinions from the users individually on how did they use this application. User1 reported that he used the application more carefully while User2 used it more casually. Besides, User2 claimed for speed mismatch for more FRR.

\subsection{Possibility of Device Sharing}

To analyze whether a trained device can be shared by some other users, we perform a different type of user testing. This testing involves two users using the application deployed in the tablet. First, the application is trained by one of the two users. Here, while the first user provides the training data, we let the second user to carefully observe him. The second user subsequently attempts to get identified as the first user in the trained application. Such attempts can be made by intruders as well as by a legitimate user. However, even after a number of attempts, the second user always gets rejected to enter the system. Afterward, the second user is guided by the first user on how to hold and swipe to mimic him. Then, the second user again starts attempting to mimic the first user. Here, after seven attempts, the second user gets succeeded. After that, the second user keeps mimicking continuously with a success rate of $40 \%$. Again, after a couple of hours break, the second user attempts to mimic the first user. However, this time the second user can not mimic the first user and fails to get identified as the first user. These results are shown in Fig. $5 \mathrm{~d}$.

These findings suggest that a trained application could be shared by others only after having a thorough knowledge and guidance. Even in such a case, the rate of acceptance of sharing is far below from the perfect value. Moreover, the sharing is completely transient, as mimicking usage is a near-to-impossible task even after a couple of hours after getting trained. This aspect guarantees a very high level of security for devices having sensitive data such as SSN, bank account number, etc.

\subsection{User Experience}

We asked a few questions to the participants after letting them use our developed application to understand the user experience. Table 3 shows the survey questions and also the summary of the responses. We can see from the responses that the majority of the users $(72 \%)$ use smart devices to store sensitive and personal information. Besides, $79 \%$ of users want to keep those information secrets where the rest are neutral about that. These two statistics confirm our motivation for devising a new mechanism for keeping the sensitive information secret in smart devices. Moreover, the majority of the users (86\%) do not disagree that our proposed system provides better security than other existing techniques such as password, pattern lock, etc. Additionally, the presented technique is easy-to-use to $79 \%$ of the participants, which proves a possible wide acceptance to the users. Finally, $43 \%$ of users are in favor of using our proposed technique in future whereas $43 \%$ remain neutral to use of the technique.

\section{APPLICATIONS OF OUR PROPOSED TECHNIQUE}

Nowadays, smart devices are frequently used to access and store highly-sensitive information. Therefore, to secure highly-sensitive information stored on these devices, users often use passwords, PINs, or pattern locks [29], which are very easy to be stolen or guessed as already discussed in this paper. In such scenarios, our proposed technique can be extremely helpful as it is near-to-impossible to mimic exhibiting up to $0 \%$ FAR, which we confirm through real experimentation in this paper. The only apparent drawback of our proposed technique is relatively high FRR (up to $39 \%$ ), which in fact suggests that for a successful entry, the user may need to swipe for multiple times. However, to ensure absolute security of highlysensitive data, this bargain could be acceptable, which is confirmed by the user experience analysis presented in earlier section.

Note that, our proposed technique can be implemented both at the kernel level and at the application level. Thus, the proposed technique can be made available to all the files stored in a device or to a set of selected ones among the files.

\section{CONCLUSION AND FUTURE WORK}

As the usage of technology is increasing day-by-day, users often face the necessity of being identified in a secured manner to protect their sensitive information. Analyzing usage data can facilitate such identification task. However, to the best of our knowledge, state-of-theart technologies in this regard have focused on usage monitoring through either in a run-time manner requiring high system overhead and resources or through incorporating password/PIN that exhibits significant vulnerability under different types of threats such as eavesdropping, shoulder surfing, etc. Hence, in this paper, we propose a single-time user identification technique utilizing touch-based and holding orientation based usage monitoring. Here, we apply various existing machine learning approaches to carry out our user identification task. Those approaches offer relatively low-accuracy having significant resource usage, which indicates the necessity of a light-weight and high-accuracy approach. Therefore, we propose a novel clustering technique named Mean-SD clustering to perform our user identification task with high accuracy incurring low resource overhead.

We perform a set of rigorous experimental evaluation to validate the efficacy of our proposed user identification technique. The experimental results indicate that our proposed technique is highly accurate in user identification. Analyzing collected data from 33 users, we find that our technique can identify users with only 5\% False Acceptance Rate and 6\% False Rejection Rate using our proposed Mean-SD clustering with a small demand on resources. Further, to demonstrate the efficacy of our proposed technique in real cases, we implement the technique in three different devices and let 27 users use the devices. Usage of the users reveals that our proposed technique offers a near-to-impossible system to breach through exhibiting as low as 0\% FAR even after making attempts through eavesdropping and shoulder surfing.

In the future, our plan is to extend our user evaluation in different states such as on the vehicle. Additionally, we plan to explore different alternatives to make our proposed Mean-SD clustering more accurate irrespective of the diversity in settings. Further, we plan to integrate our proposed technique at the kernel level of Android to enable its mass usage in the future. We envision that our proposed technique will offer a pervasive solution for mass users to protect sensitive information stored in touch-based smart devices. 


\begin{tabular}{|c|c|c|c|c|c|}
\hline Question & $\begin{array}{c}\text { Strongly } \\
\text { Agree }\end{array}$ & Agree & Disagree & $\begin{array}{l}\text { Strongly } \\
\text { Disagree }\end{array}$ & Neutral \\
\hline $\begin{array}{l}\text { I often store my sensitive and personal information (such as } \\
\text { national identification number, passport number, passwords, } \\
\text { etc.) in my smart devices. }\end{array}$ & $7 \%$ & $65 \%$ & $7 \%$ & $0 \%$ & $21 \%$ \\
\hline $\begin{array}{l}\text { I want to keep my sensitive and personal information stored } \\
\text { in my smart devices secret. }\end{array}$ & $50 \%$ & $29 \%$ & $0 \%$ & $0 \%$ & $21 \%$ \\
\hline $\begin{array}{l}\text { I am confident that the presented technique of user } \\
\text { identification can enforce the secrecy. }\end{array}$ & $14 \%$ & $15 \%$ & $7 \%$ & $7 \%$ & $57 \%$ \\
\hline $\begin{array}{l}\text { In comparison to existing techniques of user identification } \\
\text { (such as using password, pattern lock, fingerprint check, etc.), } \\
\text { the presented technique of user identification appears to be } \\
\text { more secured for keeping sensitive information secret. }\end{array}$ & $7 \%$ & $29 \%$ & $7 \%$ & $7 \%$ & $50 \%$ \\
\hline $\begin{array}{l}\text { The presented technique of user identification is easy-to-use } \\
\text { for me. }\end{array}$ & $43 \%$ & $36 \%$ & $0 \%$ & $7 \%$ & $14 \%$ \\
\hline $\begin{array}{l}\text { I will use the presented technique of user identification in } \\
\text { future. }\end{array}$ & $7 \%$ & $36 \%$ & $7 \%$ & $7 \%$ & $43 \%$ \\
\hline
\end{tabular}

Table 3: Survey questions and summary of the responses

\section{ACKNOWLEDGEMENT}

This work has been conducted in and supported by Bangladesh University of Engineering and Technology (BUET), Dhaka, Bangladesh.

\section{REFERENCES}

[1] Ildar Muslukhov, Yazan Boshmaf, Cynthia Kuo, Jonathan Lester, and Konstantin Beznosov. Understanding users' requirements for data protection in smartphones. In Data Engineering Workshops (ICDEW), 2012 IEEE 28th International Conference on, pages 228-235. IEEE, 2012.

[2] DM Hutton. Biometrics: Identity verification in a networked world. Kybernetes, 2013.

[3] Lawrence O'Gorman. Comparing passwords, tokens, and biometrics for user authentication. Proceedings of the IEEE, 91(12):2021-2040, 2003.

[4] Google Glass Snoopers Can Steal Your Passcode With a Glance. http://www.wired. com/2014/06/google-glass-snoopers-can-steal-your-passcode-with-a-glance/, 2015. [Retrieved on 14 May, 2017].

[5] Salil Prabhakar, Sharath Pankanti, and Anil K Jain. Biometric recognition: Security and privacy concerns. IEEE security \& privacy, 99(2):33-42, 2003.

[6] Tao Feng, Ziyi Liu, Kyeong-An Kwon, Weidong Shi, Bogdan Carbunar, Yifei Jiang, and Nhung Nguyen. Continuous mobile authentication using touchscreen gestures. In Homeland Security (HST), 2012 IEEE Conference on Technologies for, pages 451-456. IEEE, 2012.

[7] Mario Frank, Ralf Biedert, Eugene Ma, Ivan Martinovic, and Dawn Song. Touchalytics: On the applicability of touchscreen input as a behavioral biometric for continuous authentication. IEEE transactions on information forensics and security, 8(1):136-148, 2013.

[8] Shamir Ahmed, A S M Rizvi, Rifat Sabbir Mansur, Md Rafatul Amin, and ABM Alim Al Islam. User identification through usage analysis of electronic devices. In Networking Systems and Security (NSysS), 2015 International Conference on, pages 1-6. IEEE, 2015.

[9] Hataichanok Saevanee and Pattarasinee Bhatarakosol. User authentication using combination of behavioral biometrics over the touchpad acting like touch screen of mobile device. In Computer and Electrical Engineering, 2008. ICCEE 2008. International Conference on, pages 82-86. IEEE, 2008.

[10] Attaullah Buriro, Bruno Crispo, Filippo Del Frari, and Konrad Wrona. Touchstroke: smartphone user authentication based on touch-typing biometrics. In International Conference on Image Analysis and Processing, pages 27-34. Springer 2015.

[11] Florian Schaub, Ruben Deyhle, and Michael Weber. Password entry usability and shoulder surfing susceptibility on different smartphone platforms. In Proceedings of the 11th international conference on mobile and ubiquitous multimedia, page 13 ACM, 2012.

[12] Sébastien Marcel, Chris McCool, Cosmin Atanasoaei, Flavio Tarsetti, Jan Pesan, Pavel Matejka, Jan Cernocky, Mika Helistekangas, and Markus Turtinen. Mobio: mobile biometric face and speaker authentication. Technical report, Idiap, 2010

[13] Georgios Kambourakis, Dimitrios Damopoulos, Dimitrios Papamartzivanos, and Emmanouil Pavlidakis. Introducing touchstroke: keystroke-based authentication system for smartphones. Security and Communication Networks, 2014.

[14] Alexander De Luca, Alina Hang, Frederik Brudy, Christian Lindner, and Heinrich Hussmann. Touch me once and i know it's you!: implicit authentication based on touch screen patterns. In Proceedings of the SIGCHI Conference on Human Factors in Computing Systems, pages 987-996. ACM, 2012.

[15] Nalini K. Ratha, Jonathan H. Connell, and Ruud M. Bolle. Enhancing security and privacy in biometrics-based authentication systems. IBM systems fournal, 40 (3):614-634, 2001.

[16] Napa Sae-Bae, Kowsar Ahmed, Katherine Isbister, and Nasir Memon. Biometricrich gestures: a novel approach to authentication on multi-touch devices. In Proceedings of the SIGCHI Conference on Human Factors in Computing Systems, pages 977-986. ACM, 2012.

[17] Tao Feng, Jun Yang, Zhixian Yan, Emmanuel Munguia Tapia, and Weidong Shi. Tips: Context-aware implicit user identification using touch screen in uncontrolled environments. In Proceedings of the $15^{t h}$ Workshop on Mobile Computing Systems and Applications, page 9. ACM, 2014.

[18] Cheng Bo, Lan Zhang, Xiang-Yang Li, Qiuyuan Huang, and Yu Wang. Silentsense: silent user identification via touch and movement behavioral biometrics. In Proceedings of the $19^{\text {th }}$ annual international conference on Mobile computing \& networking, pages 187-190. ACM, 2013.

[19] Mayank Goel, Jacob Wobbrock, and Shwetak Patel. Gripsense: using built-in sensors to detect hand posture and pressure on commodity mobile phones. In Proceedings of the 25th annual ACM symposium on User interface software and technology, pages 545-554. ACM, 2012.

[20] Samsung Galaxy Young gt-s5360A. http://www.samsung.com/uk/consumer/ mobile-devices/smartphones/others/GT-S5360MAAXEU, 2017. [Retrieved on 20 January, 2017].

[21] Mobile Demographics. http://ipcarrier.blogspot.com/2009/11/ surprising-smartphone-statistics.html, 2015. [Retrieved on 14 May, 2017].

[22] Smartphone User Growth Statistics and Trends. http://brandongaille.com/ smartphone-user-growth-statistics-and-trends/, 2015. [Retrieved on 14 May, 2017].

[23] Leif E Peterson. K-nearest neighbor. Scholarpedia, 4(2):1883, 2009.

[24] S Rasoul Safavian and David Landgrebe. A survey of decision tree classifier methodology. 1990.

[25] David B Sher and Davin Milun. Generating edge detectors from a training ensemble. In Optical Engineering and Photonics in Aerospace Sensing, pages 165-176. International Society for Optics and Photonics, 1993.

[26] Samsung GALAXY Tab 10.1 LTE SC-01D. http://www.samsung.com/jp/ consumer/mobilephone/tablet/docomo/SGH-N014UWNDCM, 2017. [Retrieved on 20 January, 2017].

[27] HTC Desire 626. http://www.gsmarena.com/htc_desire_626-6968.php, 2017. [Retrieved on 20 January, 2017].

[28] Asus Zenfone 2 Laser. http://www.gsmarena.com/asus_zenfone_2_laser_ ze500kg-7478.php, 2017. [Retrieved on 14 August, 2017].

[29] How mobile device encryption works to protect sensitive data. http://searchmobilecomputing.techtarget.com/tip/ How-mobile-device-encryption-works-to-protect-sensitive-data. [Retrieved on 10 May, 2017]. 